\title{
Quantifying the effect of Vpu on the promotion of HIV-1 replication in the humanized mouse model
}

\author{
Hiroki Ikeda ${ }^{1 \dagger}$, Shinji Nakaoka ${ }^{2 \dagger}$, Rob J. de Boer ${ }^{3}$, Satoru Morita ${ }^{4}$, Naoko Misawa ${ }^{5}$, Yoshio Koyanagi ${ }^{5}$, \\ Kazuyuki Aihara ${ }^{6,7}$, Kei Sato ${ }^{5,8^{*}++}$ and Shingo Iwami $i^{1,8,9^{*}++}$
}

\begin{abstract}
Background: Tetherin is an intrinsic anti-viral factor impairing the release of nascent HIV-1 particles from infected cells. Vpu, an HIV-1 accessory protein, antagonizes the anti-viral action of tetherin. Although previous studies using in vitro cell culture systems have revealed the molecular mechanisms of the anti-viral action of tetherin and the antagonizing action of Vpu against tetherin, it still remains unclear how Vpu affects the kinetics of HIV-1 replication in vivo.

Results: To quantitatively assess the role of Vpu in viral replication in vivo, we analyzed time courses of experimental data with viral load and target cell levels in the peripheral blood of humanized mice infected with wild-type and vpudeficient HIV-1. Our recently developed mathematical model describes the acute phase of this infection reasonably, and allowed us to estimate several parameters characterizing HIV-1 infection in mice. Using a technique of Bayesian parameter estimation, we estimate distributions of the basic reproduction number of wild-type and vpu-deficient HIV1. This reveals that Vpu markedly increases the rate of viral replication in vivo.
\end{abstract}

Conclusions: Combining experiments with mathematical modeling, we provide an estimate for the contribution of Vpu to viral replication in humanized mice.

Keywords: Virus dynamics, Mathematical model, Vpu, Tetherin, HIV-1, Humanized mouse model

\section{Findings}

Tetherin is also known as bone marrow stromal antigen 2 (BST2), CD317, and HM1.24, and impairs the release of a broad range of enveloped viruses including human immunodeficiency virus type 1 (HIV-1) by tethering budded virions on the surface of infected cells [1-3]. HIV-1 expresses the accessory protein $\mathrm{Vpu}$ which antagonizes the anti-viral effect of tetherin [1-3]. Previous studies using in vitro cell culture systems have revealed the

\footnotetext{
*Correspondence: ksato@virus.kyoto-u.ac.jp; siwami@kyushu-u.org ${ }^{\dagger}$ Hiroki Ikeda, Shinji Nakaoka: Equal contribution as first author

${ }^{+\dagger}$ Kei Sato, Shingo Iwami: Equal contribution as last author

1 Department of Biology, Faculty of Sciences, Kyushu University, 6-10-1

Hakozaki, Higashi-ku, Fukuoka, Fukuoka 812-8581, Japan

${ }^{5}$ Laboratory of Viral Pathogenesis, Institute for Virus Research, Kyoto University, 53 Shogoinkawara-cho, Sakyo-ku, Kyoto, Kyoto 606-8507, Japan

Full list of author information is available at the end of the article
}

molecular mechanisms of tetherin-mediated anti-viral effect and its antagonism by $\mathrm{Vpu}$ [3-5]. We have recently performed experiments using human hematopoietic stem cell-transplanted humanized mouse model infected with wild-type and $v p u$-deficient HIV-1 (HIV-1 $v p p u$ [6, 7]). These findings suggested that Vpu counteracts the anti-viral effect of tetherin in infected humanized mice, augmenting HIV-1 replication in vivo [8]. We investigated and quantified the role of $\mathrm{Vpu}$ during the acute phase of infection using the humanized mouse model, which reasonably mimics HIV-1 infection in human before the onset of adaptive immune responses [8, 9].

To quantitatively understand the effect of $\mathrm{Vpu}$ on dynamics of viral replication and infection in vivo, we here perform mathematical modeling of the experimental data published in [8]. Previously we simplified a "basic model" of virus infection $[10,11]$, to derive a novel model 
that can be fitted to time course data from HIV-1 infected humanized mice and reliably estimate the parameters characterizing the dynamics of an acute infection (see Additional file 1) [12, 13]:

$$
\begin{aligned}
& \frac{d T(t)}{d t}=-\beta T(t) V(t), \\
& \frac{d V(t)}{d t}=r T(t) V(t)-\delta V(t) .
\end{aligned}
$$

Here $T(t)$ and $V(t)$ are the densities of target cells and virus particles, respectively, at time $t$. The parameter $\beta$ and $\delta$ are the rate constant for infection of target cells by virus and the death rate of virus producing cells, respectively. The combined parameter $r=p \beta / c$ represents the viral replication rate per target cell, where $p$ and $c$ are the virus production rate of a virus producing cell and the clearance rate of virus particles, respectively. Thanks to this simplification the model has only 5 parameters. The novel model reasonably captures de novo infection process, and these 5 parameters can be estimated more reliably than the parameters of the previous models [14-16].

To quantitatively assess the effect of Vpu on viral spread in vivo, here we used the simplified model of Eqs. $(1,2)$, and applied this to time course data of the number of $\mathrm{CD} 4^{+} \mathrm{T}$ cells per $\mathrm{ml}$ of peripheral blood $(\mathrm{PB})$ and the viral RNA load per ml of plasma of infected humanized mice [8]. We infected 9 and 10 humanized mice with CCR5-tropic wild-type (WT) HIV-1 (strain AD8) [17] and $v p u$-deficient HIV-1 (HIV-1 $\Delta v p u)[6,18]$, respectively, and $100 \mu \mathrm{l}$ of $\mathrm{PB}$ was routinely collected at $0,3,7$, 14 , and 21 days postinfection, as previously described [8, 19-21]. The amount of viral RNA in $50 \mu$ of plasma was quantified by real-time RT-PCR (Bio Medical Laboratories, Inc). Since memory $\mathrm{CD}^{+}{ }^{\mathrm{T}}$ cells are the major population of target cells, their densities were measured by hematometry and flow cytometry as previously described $[8,19-21]$. Briefly, the number of human leukocytes in $10 \mu \mathrm{l}$ of PB was measured by using a Celltac $\alpha$ MEK-6450 (Nihon Kohden, Co.), and the percentage of memory $\mathrm{CD}^{+} \mathrm{T}$ cells (i.e., $\mathrm{CD} 45^{+} \mathrm{CD}^{+} \mathrm{CD}^{+} \mathrm{CD} 45 \mathrm{RA}^{-}$cells; target cells) in human $\mathrm{CD} 45^{+}$leukocytes was analyzed by flow cytometry using a FACSCanto II (BD Biosciences).

Hereafter, we used the whole datasets from 9 WT (i.e., $v p u$-proficient) HIV-1-infected mice and 10 HIV$1 \Delta v p u$-infected mice. To assess the variability of kinetic parameters (see Additional file 1), we performed Bayesian estimation for the whole dataset using Markov Chain Monte Carlo (MCMC) sampling. To reduce the number of parameters, we allowed only the parameter $r$ to vary between the two groups, and let all other parameters be shared between WT HIV-1 and HIV-1 $1 \Delta p u$-infected mice. In addition, we allowed for broad variations in terms of the measurement error of viral load among the mouse samples into the parameter estimation via MCMC computation (i.e., the variance of the error distribution to be minimized is not constant as is typically assumed in the nonlinear least square method, c.f., [22]). The dynamics of target cells (i.e., memory $\mathrm{CD}^{+}{ }^{+} \mathrm{T}$ cells) and viral load of WT HIV-1 and HIV-1 $1 \Delta p u$ produced with the best fit parameter values are shown in Fig. 1a, c, respectively. These results revealed that the Bayesian inference works well because the model describes the acute phase of WT HIV-1 and HIV-1 $\Delta v p u$ infections in humanized mice reasonably well (c.f. $[12,13])$. The gray regions correspond to $95 \%$ posterior predictive intervals, the solid lines give the best-fit solution (mean) for Eqs. $(1,2)$, and the black and orange dots with bars show the average data with the standard deviations. We summarized the kinetic parameters estimated by the Bayesian inference in Table 1. The marginal posterior distributions for each estimated parameter are shown in Additional file 2, together with scatter plots of paired parameters. Although the ranges of these posterior distributions were relatively narrows, they were not identifiable because $r, \delta$, and $T(0)$ correlate with one another. We also fitted our model to the individual data from each of the 9 and 10 humanized mice infected with WT HIV-1 and HIV$1 \triangle v p u$, respectively [using the FindMinimum package of Mathematica 9.0 to minimize the sum of squared residuals (see Additional files 2, 3, 5)]. Not surprisingly, this revealed that the two methods gave very consistent estimates for the parameters underlying WT HIV-1 and HIV-1 $\Delta v p u$ infection in humanized mice.

To reduce the effects of the limited identifiability of $r$, $\delta$, and $T(0)$, we combined them into a single parameter $R_{0}$. The basic reproductive number $R_{0}=r T(0) / \delta$ is a well known quantity which is defined as the average number of newly infected cells produced from any one infected cell, under conditions where most of the target cells are uninfected $[12,13]$. For the mice which had enough data to estimate the death rate of infected cells, we directly calculated $R_{0}$ using individual estimation of $T(0), r$ and $\delta$. Additionally, we calculated $R_{0}$ using the previously estimated mean death rate of $\delta=0.6$ per day (see Additional files $3,4,5)$. The average basic reproductive number of WT HIV-1 and HIV-1 $1 \Delta v p u$ in humanized mice is $R_{0}=2.09 \pm 0.78$ (mean \pm standard deviation) and $1.72 \pm 0.35$, respectively. Interestingly, although $v p u$ is not an essential gene for HIV-1 replication [8], we found that the average of the estimated $R_{0}$ of WT HIV-1 tends to be somewhat greater than that of HIV-1 $\Delta v p u$. We used all accepted MCMC parameter estimates from the whole datasets, and calculated that the mean values and the $95 \%$ CIs of $R_{0}$ for WT HIV-1 and HIV-1 $1 \Delta v p u$ are 2.43 (95 \% CI 1.78-3.26) and 2.25 (95 \% CI 1.36-3.76), 

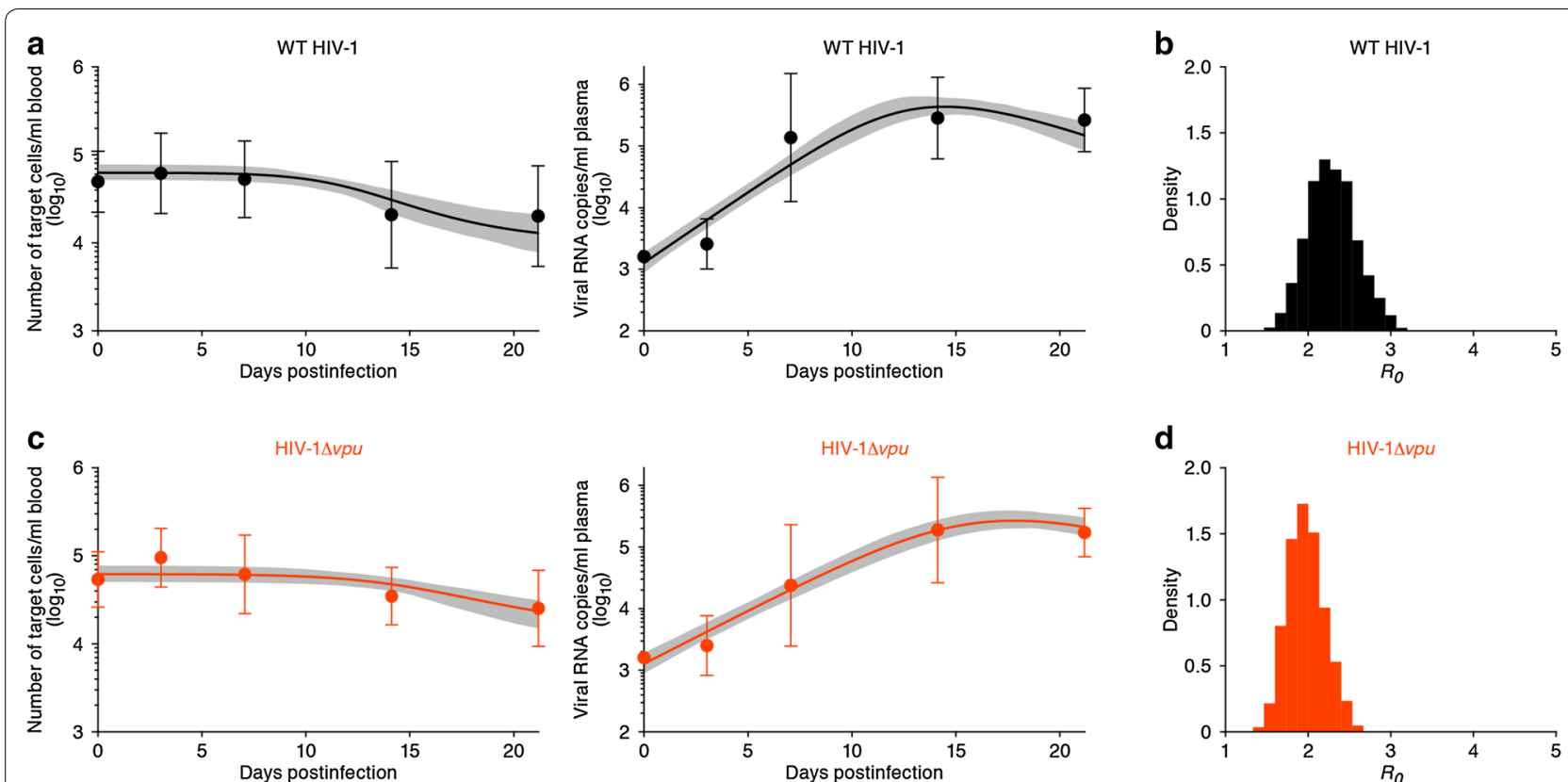

Fig. 1 Variability of virus dynamics and basic reproduction number in HIV-1 and HIV-1 $\triangle v p u$ infected humanized mice. The predicted variability of the dynamics of target cells (left) and viral loads (right) of WT HIV-1 (a) and HIV-1 $\triangle v p u$ (c) are shown based on Bayesian estimation for the whole datasets using MCMC sampling. The gray regions correspond to $95 \%$ posterior predictive intervals. The solid lines give the best-fit solution for Eqs. (1, 2), and the bullets with error bars show the average with standard deviations. Note that the initial viral loads are set at the detection limit for all samples. The distributions of calculated $R_{0}$ from all accepted MCMC parameter estimates for WT HIV-1 and HIV-1 $\triangle v p u$ are shown in $\mathbf{b}$ and $\mathbf{d}$, respectively. For each plot, the last 7000 MCMC samples among the total 10,000 samples are used

Table 1 Parameters values and derived quantities for the humanized mouse experiments by the Bayesian inference

\begin{tabular}{|c|c|c|c|c|c|c|}
\hline $\begin{array}{l}\text { Strain [best fit } \\
\text { value }(95 \% \mathrm{Cl}) \text { ] }\end{array}$ & $T(0)($ cells $/ \mathrm{ml})$ & $\begin{array}{l}V(0) \text { (RNA copies/ } \\
\mathrm{ml})\end{array}$ & $\begin{array}{l}\beta[(\text { virion/ } \\
\mathrm{ml}^{-1} \text { day }^{-1} \\
\left.\left(\times 10^{-7}\right)\right]\end{array}$ & $\delta\left(\right.$ day $\left.^{-1}\right)$ & $\begin{array}{l}r^{\dagger}\left[(\text { cell/ml })^{-1} \text { day }^{-1}\right. \\
\left.\left(\times 10^{-6}\right)\right]\end{array}$ & $R_{0}^{\ddagger}(-)$ \\
\hline WT HIV-1 & $84,621(66,301-105,897)$ & $1324(925-1837)$ & $4.41(2.58-6.78)$ & $0.428(0.261-0.699)$ & 11.50 (7.79-16.84) & $2.29(1.75-2.90)$ \\
\hline HIV-1 $\Delta v p u$ & & & & & $9.97(6.59-15.06)$ & $1.97(1.58-2.44)$ \\
\hline
\end{tabular}

${ }^{\dagger} p=0.138$ by the repeated bootstrap $t$ test and Cohen's $d=0.664$ (statistical power $=0.290$ ) between WT HIV-1 and HIV-1 $\Delta v p u$. 10 parameter sets are sampled from the posterior predictive distribution

${ }^{\ddagger} p=0.035$ by the repeated bootstrap $t$ test and Cohen's $d=1.395$ (statistical power $=0.839$ ) between WT HIV- 1 and HIV-1 $\Delta v p u$. 10 parameter sets are sampled from the posterior predictive distribution

respectively (see Table 1). The distributions of calculated $R_{0}$ for WT HIV-1 and HIV-1 $\Delta v p u$ are shown in Fig. 1b, $\mathrm{d}$, respectively. Despite the small difference, the mean value of $R_{0}$ for WT HIV-1 is significantly larger than that of that of HIV-1 $1 \Delta v p u(P<0.01$ by the repeated bootstrap $t$ test).

The retention of virion anchored by tetherin on the surface of infected cells should be reflected by the virus production rate, $p$, in the basic model (see $[10,11])$. In addition to its effects on tetherin, Vpu degrades CD4 molecules on the surface of infected cells, and this function of Vpu is highly conserved in pandemic HIV-1 [4, 23-25]. The degradation of the viral receptor CD4 likely prevents aberrant interactions between newly synthesized CD4 molecules and novel viral envelope glycoproteins in the endoplasmic reticulum of infected cells [26-29]. Hence $\mathrm{Vpu}$ is expected to increase the number of infectious particles produced per infected cell. We have recently shown that Vpu is closely associated with the down-modulation of CD4 molecules from the infected cells during the acute phase of infection in vivo [8]. Therefore, one could argue that the infection rate $\beta$ in the basic model also decreases in the absence of $\mathrm{Vpu}$. It remains unclear whether this additional effect of Vpu plays a major quantitative role, but fortunately both effects are combined in the composed parameter $r=p \beta / c$ of our model. Hence, the difference in the estimated parameters, especially for the basic reproduction number $\left(R_{0}=r T(0) / \delta\right)$, between 
WT HIV-1 and HIV-1 $1 \Delta p u$ infection reflects the overall role of Vpu in humanized mouse model.

In a previous study we compared the viral loads of WT HIV-1 with HIV-1 $1 \Delta p u$ at different time points in bone marrow (BM) and spleen [8], and observed differences in the viral load only at the earliest time point in the spleen. The amount of cell-free virions in spleen is 100-10,000 fold higher than that in BM [8]. These results suggest that the main source of viral particles in infected humanized mice is the spleen. This is in good agreement with the fact that we now establish that these two viruses differ in the replication rate, allowing a WT virus to approach the set-point viral load in the spleen at an earlier point in time. It could be that the set-point viral load is not strongly dependent on the replication rate (as set-points are also determined by innate immune responses and target cell availability), or that during chronic infection the virus replicates by cell-to-cell infection, which depends less on tetherin [8]. In addition, it is well known that the main target of HIV-1 infection is human $\mathrm{CD} 4^{+} \mathrm{T}$ cells and monocytes/macrophages. In this regard, we have previously reported that human $\mathrm{CD} 4^{+} \mathrm{T}$ cells are relatively frequent in spleen, while monocytes/macrophages are more frequent in BM [19]. Since the current data were collected from the blood, we still do not understand why the approach to set-point is faster in the BM than in the spleen, in terms of kinetics. The evaluation of tissuespecific effects of $\mathrm{Vpu} /$ tetherin on viral pathogenesis/replication requires further investigation, e.g., using a spatial model of viral replication [30].

To the best of our knowledge, this is the first report quantifying the role of Vpu in viral spread in vivo. Our findings suggest that the ability of Vpu to down-regulate both tetherin and CD4 moderately increases HIV-1 replication during acute infection. Since the initial viral replicative capacity plays an important role in the subsequent course of disease [31], this moderate increase could still have a large effect on the progression to AIDS.

\section{Additional files}

Additional file 1. Supplementary Materials and Methods. Fitting model to time course experimental data.

Additional file 2. Supplementary Figures. Posterior distributions for each estimated parameter with pairwise scatter plots.

Additional file 3. Supplementary Results. Parameter estimation for individual data from infected humanized mouse with WT HIV-1 and HIV-1 1 vvpu.

Additional file 4. Figures for Supplementary Results. Dynamics of WT HIV-1 and HIV-1 $\triangle$ vpu infections in humanized mice.

Additional file 5. Tables for Supplementary Results. Parameters values, initial values and derived quantities for the humanized mice infected with the WT HIV-1 and HIV-1 $\triangle$ vpu.

\section{Abbreviations}

HIV-1: human immunodeficiency virus type-1; Vpu: viral protein U; HIV-1 $\Delta$ vpu: vpu-deficient HIV-1; PB: peripheral blood; MCMC: Markov Chain Monte Carlo.

\section{Authors' contributions}

YK, KA, KS and SI designed the study. HI, SN, RDB, SM and SI carried out data analysis. NM, YK and KS performed all experiments. HI, SN, RDB, SM, KA and SI developed mathematical model. HI, SN, RDB, YK, KA, KS and SI wrote the final manuscript. All authors read and approved the final manuscript.

\section{Author details}

${ }^{1}$ Department of Biology, Faculty of Sciences, Kyushu University, 6-10-1 Hakozaki, Higashi-ku, Fukuoka, Fukuoka 812-8581, Japan. ${ }^{2}$ Graduate School of Medicine, University of Tokyo, Tokyo, Japan. ${ }^{3}$ Theoretical Biology, Utrecht University, Utrecht, The Netherlands. ${ }^{4}$ Department of Mathematical and Systems Engineering, Shizuoka University, Shizuoka, Japan. ${ }^{5}$ Laboratory of Viral Pathogenesis, Institute for Virus Research, Kyoto University, 53 Shogoinkawara-cho, Sakyo-ku, Kyoto, Kyoto 606-8507, Japan. ${ }^{6}$ Institute of Industrial Science, The University of Tokyo, Tokyo, Japan. ${ }^{7}$ Graduate School of Information Science and Technology, The University of Tokyo, Tokyo, Japan. ${ }^{8}$ CREST, JST, Saitama, Japan. ${ }^{9}$ PRESTO, JST, Saitama, Japan.

\section{Acknowledgements}

This research is in-part supported by Kyushu University Fund and Grant-inAid for JSPS Fellows (to H.I.); Grants-in-Aid for Young Scientists B25871132 (to S.N.), B23790500 (to K.S.) and B258000092 (to S.I.) from the Japan Society for the Promotion of Science (JSPS); the Japan Agency for Medical Research and Development, AMED (H27-ShinkoJitsuyoka-General-016) (to S.N., K.S., and S.I.); the Aihara Innovative Mathematical Modeling Project, JSPS, through the "Funding Program for World-Leading Innovative R \& D on Science and Technology (FIRST Program)", initiated by Council for Science and Technology Policy (to S.I., S.N., K.A., and K.S.); Takeda Science Foundation (to K.S.); the Shimizu Foundation for Immunological Research Grant (to K.S.); Sumitomo Foundation Research Grant (to K.S.); Senshin Medical Research Foundation (to K.S.); Imai Memorial Trust for AIDS Research (to K.S.); Ichiro Kanehara Foundation (to K.S.); Kanae Foundation for the Promotion of Medical Science (to K.S.); Suzuken Memorial Foundation (to K.S.); the Uehara Memorial Foundation (to K.S.); JST CREST program (to S.M., K.S. and S.I.); JST PRESTO program (to S.I.), JSPS KAKENHI Grant Number 10192783 and 15 KT0107 (to S.I.), with additional funding from the Inamori Foundation (to S.I.).

\section{Competing interests}

The authors declare that they have no competing interests.

Received: 28 October 2015 Accepted: 15 March 2016

Published online: 18 April 2016

\section{References}

1. Van Damme N, Goff D, Katsura C, Jorgenson RL, Mitchell R, Johnson MC, Stephens EB, Guatelli J. The interferon-induced protein BST-2 restricts HIV-1 release and is downregulated from the cell surface by the viral Vpu protein. Cell Host Microbe. 2008;3:245-52.

2. Neil SJ, Zang T, Bieniasz PD. Tetherin inhibits retrovirus release and is antagonized by HIV-1 Vpu. Nature. 2008;451:425-30.

3. Sato K, Gee P, Koyanagi Y. Vpu and BST2: still not there yet? Front Microbiol. 2012;3:131.

4. Kirchhoff F. Immune evasion and counteraction of restriction factors by HIV-1 and other primate lentiviruses. Cell Host Microbe. 2010;8:55-67.

5. Sauter D. Counteraction of the multifunctional restriction factor tetherin. Front Microbiol. 2014;5:163.

6. Schubert U, Clouse KA, Strebel K. Augmentation of virus secretion by the human immunodeficiency virus type $1 \mathrm{Vpu}$ protein is cell type independent and occurs in cultured human primary macrophages and lymphocytes. J Virol. 1995;69:7699-711.

7. Schubert U, Bour S, Willey RL, Strebel K. Regulation of virus release by the macrophage-tropic human immunodeficiency virus type 1 AD8 isolate is redundant and can be controlled by either Vpu or Env. JVirol. 1999;73:887-96. 
8. Sato K, Misawa N, Fukuhara M, Iwami S, An DS, Ito M, Koyanagi Y. Vpu augments the initial burst phase of HIV-1 propagation and downregulates BST2 and CD4 in humanized mice. J Virol. 2012;86:5000-13.

9. Sato K, Misawa N, Iwami S, Satou Y, Matsuoka M, Ishizaka Y, Ito M, Aihara K, An DS, Koyanagi Y. HIV-1 Vpr accelerates viral replication during acute infection by exploitation of proliferating $\mathrm{CD}^{+}{ }^{+} \mathrm{T}$ cells in vivo. PLoS Pathog. 2013;9:e1003812

10. Perelson AS. Modelling viral and immune system dynamics. Nat Rev Immunol. 2002;2:28-36.

11. Nowak M, May RM. Virus dynamics: mathematical principles of immunology and virology: mathematical principles of immunology and virology. Oxford: Oxford University Press; 2000

12. Ikeda H, de Boer RJ, Sato K, Morita S, Misawa N, Koyanagi Y, Aihara K, Iwami S. Improving the estimation of the death rate of infected cells from time course data during the acute phase of virus infections: application to acute HIV-1 infection in a humanized mouse model. Theor Biol Med Model. 2014;11:22.

13. Ikeda H, Nakaoka S, Sato K, Misawa N, Koyanagi Y, Iwami S. Effect of eclipse phase on quantifying viral dynamics of acute HIV-1 infection in humanized mouse model. Nonlinear Theory Appl IEICE. 2015;6:47-53.

14. Little SJ, McLean AR, Spina CA, Richman DD, Havlir DV. Viral dynamics of acute HIV-1 infection. J Exp Med. 1999;190:841-50.

15. Ribeiro RM, Qin L, Chavez LL, Li D, Self SG, Perelson AS. Estimation of the initial viral growth rate and basic reproductive number during acute HIV-1 infection. JVirol. 2010;84:6096-102.

16. Nowak MA, Lloyd AL, Vasquez GM, Wiltrout TA, Wahl LM, Bischofberger N, Williams J, Kinter A, Fauci AS, Hirsch VM, Lifson JD. Viral dynamics of primary viremia and antiretroviral therapy in simian immunodeficiency virus infection. J Virol. 1997;71:7518-25.

17. Theodore TS, Englund G, Buckler-White A, Buckler CE, Martin MA, Peden KW. Construction and characterization of a stable full-length macrophage-tropic HIV type 1 molecular clone that directs the production of high titers of progeny virions. AIDS Res Hum Retrovir. 1996;12:191-4.

18. Stafford MA, Corey L, Cao Y, Daar ES, Ho DD, Perelson AS. Modeling plasma virus concentration during primary HIV infection. J Theor Biol. 2000;203:285-301.

19. Nie C, Sato K, Misawa N, Kitayama H, Fujino H, Hiramatsu H, Heike T, Nakahata T, Tanaka Y, Ito M, Koyanagi Y. Selective infection of CD4+ effector memory $T$ lymphocytes leads to preferential depletion of memory $T$ lymphocytes in R5 HIV-1-infected humanized NOD/SCID/IL-2Ry null mice. Virology. 2009;394:64-72.
20. Sato K, Takeuchi JS, Misawa N, Izumi T, Kobayashi T, Kimura Y, Iwami S, Takaori-Kondo A, Hu WS, Aihara K, et al. APOBEC3D and APOBEC3F potently promote HIV-1 diversification and evolution in humanized mouse model. PLoS Pathog. 2014;10:e1004453.

21. Sato K, Izumi T, Misawa N, Kobayashi T, Yamashita Y, Ohmichi M, Ito M, Takaori-Kondo A, Koyanagi Y. Remarkable lethal G-to-A mutations in vifproficient HIV-1 provirus by individual APOBEC3 proteins in humanized mice. J Virol. 2010;84:9546-56.

22. Soetaert K, Petzoldt T. Inverse modelling, sensitivity and monte carlo analysis in R using package FME. J Stat Softw. 2010;33.

23. Bour S, Schubert U, Strebel K. The human immunodeficiency virus type Vpu protein specifically binds to the cytoplasmic domain of CD4: implications for the mechanism of degradation. J Virol. 1995;69:1510-20.

24. Sauter D, Schindler M, Specht A, Landford WN, Munch J, Kim KA, Votteler $J$, Schubert U, Bibollet-Ruche F, Keele BF, et al. Tetherin-driven adaptation of Vpu and Nef function and the evolution of pandemic and nonpandemic HIV-1 strains. Cell Host Microbe. 2009;6:409-21.

25. Iwami S, Sato K, Morita S, Inaba H, Kobayashi T, Takeuchi JS, Kimura Y, Misawa N, Ren F, Iwasa Y, et al. Pandemic HIV-1 Vpu overcomes intrinsic herd immunity mediated by tetherin. Sci Rep. 2015;5:12256

26. Willey RL, Maldarelli F, Martin MA, Strebel K. Human immunodeficiency virus type 1 Vpu protein induces rapid degradation of CD4. J Virol. 1992;66:7193-200.

27. Margottin F, Bour SP, Durand H, Selig L, Benichou S, Richard V, Thomas D, Strebel K, Benarous R. A novel human WD protein, h-beta $\operatorname{TrCp}$, that interacts with HIV-1 Vpu connects CD4 to the ER degradation pathway through an F-box motif. Mol Cell. 1998;1:565-74.

28. Schubert U, Anton LC, Bacik I, Cox JH, Bour S, Bennink JR, Orlowski M, Strebel K, Yewdell JW. CD4 glycoprotein degradation induced by human immunodeficiency virus type $1 \mathrm{Vpu}$ protein requires the function of proteasomes and the ubiquitin-conjugating pathway. J Virol. 1998;72:2280-8.

29. Levesque K, Finzi A, Binette J, Cohen EA. Role of CD4 receptor downregulation during HIV-1 infection. Curr HIV Res. 2004;2:51-9.

30. Lorenzo-Redondo R, Fryer HR, Bedford T, Kim EY, Archer J, Pond SLK, Chung YS, Penugonda S, Chipman JG, Fletcher CV, et al. Persistent HIV-1 replication maintains the tissue reservoir during therapy. Nature. 2016;530:51-6.

31. Claiborne DT, Prince JL, Scully E, Macharia G, Micci L, Lawson B, Kopycinski J, Deymier MJ, Vanderford TH, Nganou-Makamdop K, et al. Replicative fitness of transmitted HIV-1 drives acute immune activation, proviral load in memory $\mathrm{CD}^{+}{ }^{+} \mathrm{T}$ cells, and disease progression. Proc Natl Acad Sci USA. 2015;112:E1480-9.

\section{Submit your next manuscript to BioMed Central and we will help you at every step:}

- We accept pre-submission inquiries

- Our selector tool helps you to find the most relevant journal

- We provide round the clock customer support

- Convenient online submission

- Thorough peer review

- Inclusion in PubMed and all major indexing services

- Maximum visibility for your research

Submit your manuscript at www.biomedcentral.com/submit
() Biomed Central 